Case Report

\title{
Dentigerous Cyst or Adenomatoid Odontogenic Tumor: Clinical Radiological and Histopathological Dilemma
}

\author{
Shivesh Acharya, ${ }^{1}$ Ashima Goyal, ${ }^{1}$ Vidya Rattan, ${ }^{2}$ Kim Vaiphei, ${ }^{3}$ and Sarabjot Kaur Bhatia \\ ${ }^{1}$ Department of Pediatric and Preventive Dentistry, Oral Health Sciences Center, \\ Postgraduate Institute of Medical Education and Research, Chandigarh 160012, India \\ ${ }^{2}$ Department of Oral and Maxillofacial Surgery, Oral Health Sciences Center, \\ Postgraduate Institute of Medical Education and Research, Chandigarh 160012, India \\ ${ }^{3}$ Department of Histopathology, Postgraduate Institute of Medical Education and Research, Chandigarh 160012, India
}

Correspondence should be addressed to Shivesh Acharya; drshivesh3@yahoo.com

Received 28 February 2014; Revised 18 May 2014; Accepted 4 June 2014; Published 1 July 2014

Academic Editor: Martin G. Mack

Copyright (C) 2014 Shivesh Acharya et al. This is an open access article distributed under the Creative Commons Attribution License, which permits unrestricted use, distribution, and reproduction in any medium, provided the original work is properly cited.

Adenomatoid odontogenic tumor (AOT) is a well-recognised slow growing benign tumor derived from complex system of dental lamina or its remnants. This lesion is categorised into three variants of which the more common variant is follicular type which is often mistaken for dentigerous cyst. We present a case of AOT in a 14-year-old male who was misdiagnosed as dentigerous cyst. Clinical radiological and therapeutic characteristics of the case are commented on in detail.

\section{Introduction}

Adenomatoid odontogenic tumor (AOT) was first described by Ghosh in 1934 [1] as an adamantinoma of the maxilla and was first recognised as distinct entity by Stafne in 1948 [2]. Later on it has been described under various names like adenoameloblastoma, cystic complex composite odontoma, ameloblastic odontogenic tumor, odontogenic adenomatoid tumor, and so forth. WHO in 1971 adopted the term proposed by Philipsen and Birn [3] as AOT and defined lesion as "a tumor of odontogenic epithelium with duct-like structures and with varying degrees of inductive change in the connective tissue. The tumor may be partially cystic, and in some cases solid lesion may be present as masses in the wall of large cyst. It is believed that lesion is not a neoplasm" [4]. Philipsen et al. subdivided this condition into three groups referred to as follicular, extrafollicular, and peripheral. These variants have common histologic characteristics that indicate a common origin as derived from the complex system of dental lamina or its remnant [5]. The follicular and extrafollicular variants account for $96 \%$ of all AOT and of these $71 \%$ are follicular variants. The peripheral variant is the rarest with only 18 cases reported so far [6]. The follicular variant is predominantly associated with the crown and often part of the root of an impacted (unerupted) tooth. The most frequently associated tooth is the maxillary canine rarely the permanent molars. Based on the clinical and radiographic examination follicular variant is often initially mistaken as dentigerous cyst [3]. Here we present a case of AOT which presented as cyst like lesion around the crown of unerupted maxillary canine and was initially mistaken as dentigerous cyst.

\section{Case Report}

A 14-year-old boy presented to the Unit of Pedodontics and Preventive Dentistry, Oral Health Sciences Centre, PGIMER, Chandigarh, India, with swelling and pain on right side of the face. The detailed history reported by the father revealed that they first noticed the swelling 4-5 months back. The swelling was progressively increasing in size and strawcolored fluid occasionally exuded from the swelling. A private dental practitioner was consulted for the same who extracted maxillary right primary canine and first molar. The diagnosis of infected cyst (dentigerous) was made histopathologically. 


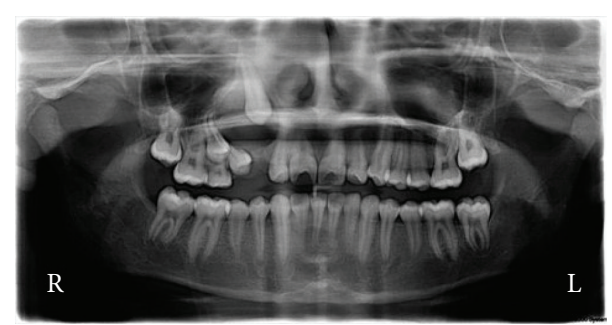

FIGURE 1: Preoperative orthopantomograph showing radiolucent lesion in relation to unerupted right maxillary canine.

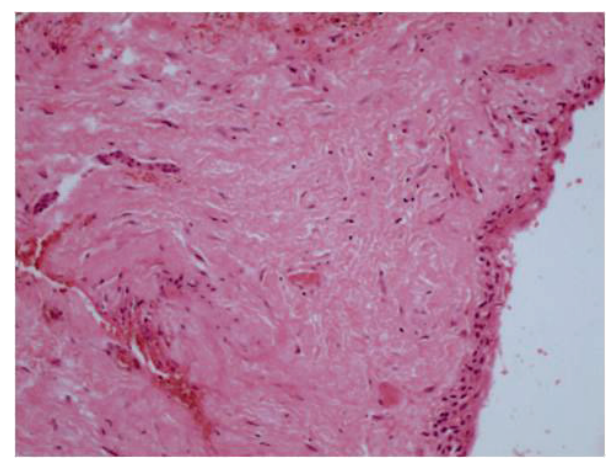

FIGURE 2: Focus showing fragments lined by squamous epithelium with wall composed of fibrocollagenous tissue consistent with dentigerous cyst (hematoxylin and eosin 10x).

Swelling persisted one month after treatment so the patient was referred to Oral Health Sciences Centre, PGIMER, Chandigarh. At clinical examination on initial visit an extraoral facial swelling approximately measuring $1 \times 3 \mathrm{~cm}$ was noted on right side of the face obliterating the nasolabial fold. Swelling was tender and fluctuant with well-defined margins. Intraoral examination revealed permanent dentition with missing permanent canine, first and second premolar, and swelling extending from right maxillary lateral incisor to first premolar region. Orthopantomograph revealed a welldefined radiolucent lesion in relation to unerupted maxillary right permanent canine, first premolar, and second premolar, extending from distal surface of lateral incisor to mesial surface of first premolar and which has also caused the displacement of roots of the adjacent lateral incisor and the first premolar (Figure 1).

On diagnostic aspiration, straw-colored fluid was drawn from the lesion. Based on clinical and radiographic evaluation a provisional diagnosis of dentigerous cyst was made and conservative approach was planned to marsupialize the cystic swelling. Swelling was marsupialized under local anesthesia and an acrylic stent was positioned to maintain patency and to allow for eruption of permanent canine. Part of cystic lining was evaluated histopathologically which showed fragments lined by squamous epithelium with wall composed of fibrocollagenous tissue with minimum inflammation features consistent with dentigerous cyst (Figure 2).

The patient was put on antibiotics and analgesics for 5 days and was followed up on monthly basis. The patient was

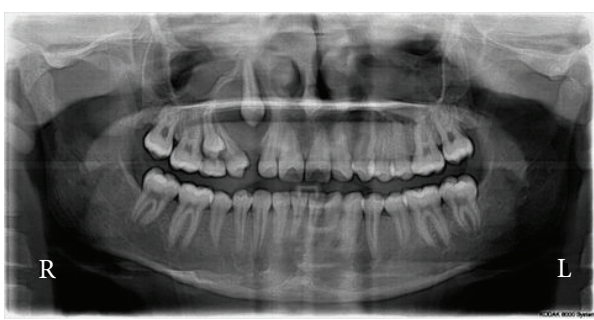

FIGURE 3: Panoramic radiograph taken after marsupialization of the cyst showing no movement of the canine.

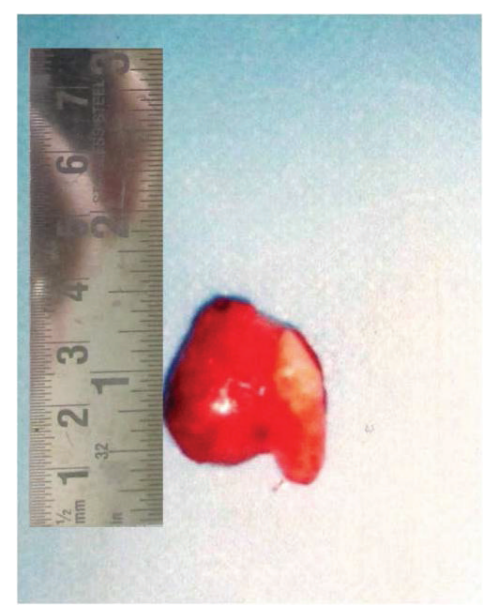

FIgURE 4: Canine along with the lesion in toto removed surgically.

reinforced to maintain oral hygiene on each follow-up and was evaluated for eruption of canine radiographically. At 2month follow-up visit, occasional discharge from the swelling was present and detailed evaluation of panoramic radiograph (Figure 3) showed no eruptive movement in canine and radiolucent area was still persisting.

At this stage a decision to surgically remove the canine along with removal of the lesion in toto was taken. The lesion was completely enucleated under local anaesthesia along with permanent canine. The cyst was separated easily from the adjoining bone and there was no evidence of oronasal and oroantral communication and the palatal mucosa was intact. The wound was then sutured closed. The surgical specimen enveloping a permanent tooth was smooth and reddish in color and measured approximately $20 \times 20 \times 15 \mathrm{~mm}$ (Figure 4).

The surgical specimen was submitted for histopathological examination. Histopathology report revealed solid proliferation of polygonal and spindle shaped cells with only scanty stroma of connective tissue associated with ductlike and rosette-like structures. Deposition of eosinophilic homogenous material within the rosette-like structures was also seen. (Figure 5).

Patient was followed up regularly and no evidence of any discharge or recurrence of swelling was noted. Orthopantomograph taken at three-month follow-up showed sign of resolution of radiolucency. The patient was advised to undergo multibracketed treatment for space closure. At 


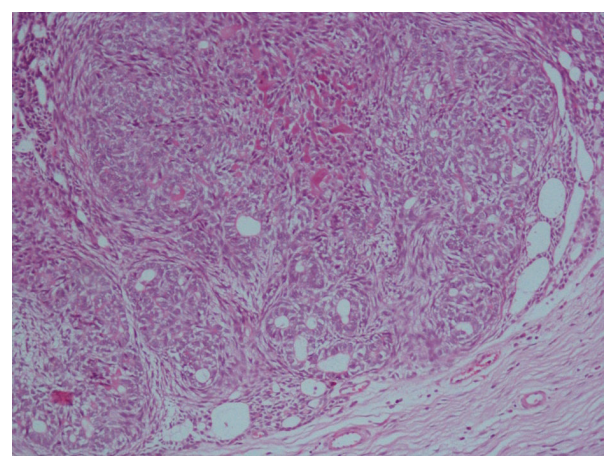

FIGURE 5: Solid proliferation of polygonal and spindle shaped cells with only scanty stroma of connective tissue associated with duct-like and rosette-like structures suggestive of AOT (hematoxylin and eosin stain 40x).

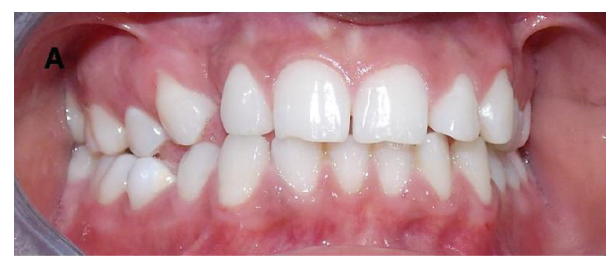

(a)

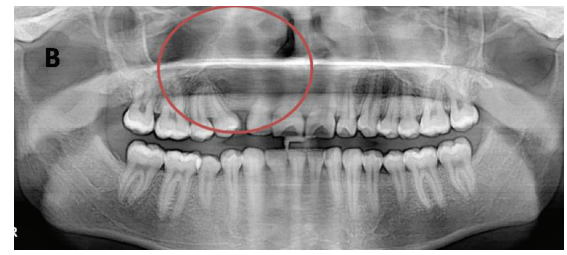

(b)

FIgURE 6: (a) Clinical presentation at 3-year follow-up. (b) Orthopantomograph taken at three- year follow-up.

three-year follow-up, there was no evidence of any discharge or recurrence (Figure 6(a)) and panoramic radiograph revealed normal bone healing (Figure 6(b)).

\section{Discussion}

An extensive review of 500 cases of AOT has been conducted by Philipsen et al. [6]. Leon et al. described a multicentre study of both the clinicopathological and immunohistochemical features of 39 cases of AOT. Two-third of these were diagnosed in the second decade of life, and over $50 \%$ occurred in adolescents between ages of 13 and 19 [14].

Our patient falls into this group, but it is noted that range of occurrence is very wide (3-82 years). Both follicular and extrafollicular variants occur more commonly in the maxilla than in the mandible, with a ratio of $2.1: 1$. The female: male ratio for all age groups and AOT variants together is $2: 1$, with an even higher female preponderance (approximately $3: 1$ ) among certain Asian populations $[5,14]$. Our patient is an Asian male. Cystic presentation of AOT has been reported way back in 1915 by Harbitz who reported the lesion as "cystic Adamantoma" [15]. The most common presentation of AOT radiologically is the unilocular cystic mass enclosing the unerupted tooth (the reason it is commonly taken as a dentigerous cyst). Also, histopathologically, the lesion may rarely present with a cystic component. Only recently the cystic nature of AOT has been in debate. The bisected lesion may show varying degrees of cystic change and rarely the tumor may entirely be cystic [16]. The systematic review of the literature of AOTs associated with or originating from an odontogenic cyst has been conducted by Gadewar et al. [16]. The cystic component of AOT has been variedly termed as dentigerous cyst $[9,10,13]$, calcifying odontogenic cyst $[17,18]$, or unilocular ameloblastoma [19]. However, in pediatric population very few cases have been described that arise in association with a dentigerous cyst. A systematic search of the English language medical literature revealed only seven such cases in children and adolescents in the age range of $8-18$ years (PubMed search using the key words adenomatoid odontogenic tumour, dentigerous cyst). The clinical characteristics of these cases and the current case are summarised in Table 1.

It is noted that the male to female ratio is $7: 1$ and nearly all the cases occurred during the second decade of life except one which is reported in 8-year-old male child. Most of the lesions appeared as a well-circumscribed unilocular radiolucency around the unerupted tooth and the most commonly involved tooth was the maxillary canine (6 cases).

The origin of the AOT is controversial. Some have focused on the idea that its origin is from the odontogenic epithelium of the dentigerous cyst, while others believe that tumors could be derived from epithelial remnants of the dental lamina complex system. The lesion grows into a nearby dental follicle or next to follicle leading to "envelopmental" theory [20]. Chen et al. even suggested the term "hybrid variant" where AOT is derived from dentigerous cyst. In our case the tumor surrounded the fully formed canine suggesting an envelopmental pathogenesis or "hybrid variant" [12].

The interest and relevance of the present case are the difficulty to diagnose accurately based on the radiograph and histopathology. The initial histopathological report in the present case stated findings suggestive of dentigerous cyst and later report suggested findings corresponding to 
TABLE 1: Clinical data of the reported cases of adenomatoid odontogenic tumor (AOT) arising from a dentigerous cyst in children and adolescents.

\begin{tabular}{lccccc}
\hline Reference & Age/Sex & Race & Radiographic & Features \\
\hline Valderrama [7] & 16 females & Filipino & Unilocular radiolucency & 14 crown surrounded & Maxilla \\
Warter et al. [8] & 8 males & Nigerian & Unilocular radiolucency & 13 crown surrounded & Maxilla \\
Tajima et al. [9] & 15 males & Japanese & A well-defined radiopaque mass & Crown of unerupted 28 & Maxillary sinus \\
Garcia-pola et al. [10] & 12 males & Spanish & Unilocular radiolucency & 23 crown & Maxilla \\
Bravo et al. [11] & 14 males & Not stated & Unilocular radiolucency & 23 crown surrounded & Maxilla \\
Chen et al. [12] & 18 males & Chinese & Unilocular radiolucency & 23 crown surrounded & Maxilla \\
Nonaka et al. [13] & 13 males & Not stated & Unilocular radiolucency & 23 crown & 13 crown \\
Present case & 14 males & Asian & Unilocular radiolucency & Maxilla & Maxilla \\
\hline
\end{tabular}

those of adenomatoid odontogenic tumor. Whether it was dentigerous cyst transforming to adenomatoid tumor or a cystic variant of adenomatoid odontogenic tumor in the present case could not be stated with exactitude as initially to preserve associated tooth only part of cystic lining was removed for histopathological evaluation. Gadewar et al. [16] suggested that incisional biopsy depicting the cystic lining alone would inaccurately identify the lesion as dentigerous cyst or unicystic ameloblastoma. The use of MRI and particularly dynamic contrast enhanced MRI to distinguish AOT from other odontogenic lesions that have been described [21].

Both dentigerous cyst and adenomatoid odontogenic tumors are entirely benign, encapsulated lesions, and enucleation poses no major difficulties. If the dental follicle is found to be uninvolved during surgery and if it can be easily separated from the tumor, it may be possible to remove the lesion while leaving the teeth in place [22]. In the present case report permanent canine was embedded in the tumor, and the large size and close approximation of the lesion to the erupted teeth made it impossible to save the tooth. No aggressive behaviour on the part of the adenomatoid tumors has been described, and recurrence is very rare following correct enucleation of the primary lesion [23].

\section{Conclusion}

As depicted in the present case, AOT is often mistaken as dentigerous cyst radiologically as well as histopathologically, and in that context even in pediatric population few case reports of AOT arising from or associated with dentigerous cyst have been reported. However, the present case highlights the importance of the fact that in cases of unilocular lesion surrounding the impacted tooth in the anterior maxillary region the treatment as per AOT should be followed.

\section{Conflict of Interests}

The authors declare that there is no conflict of interests regarding the publication of this paper.

\section{References}

[1] L. S. Ghosh, "Adamantinoma of the upper jaw," The American Journal of Pathology, vol. 10, pp. 773-389, 1934.
[2] E. C. Stafne, "Epithelial tumors associated with developmental cysts of the maxilla: a report of three cases," Oral Surgery, Oral Medicine, Oral Pathology, vol. 1, no. 10, pp. 887-894, 1948.

[3] H. P. Philipsen and H. Birn, "The adenomatoid odontogenic tumour, ameloblastic adenomatoid tumour or adenoameloblastoma," Acta Pathologica et Microbiologica Scandinavica, vol. 75, no. 3, pp. 375-398, 1969.

[4] P. A. Reichart and H. P. Philipsen, "Adenomatoid odontogenic tumour," in Odontogenic Tumors and Allied Lesions, P. A. Reichart, P. Reichart, and H. P. Philipsen, Eds., pp. 105-116, Quintessence, London, UK, 2004.

[5] H. P. Philipsen, P. A. Reichart, K. H. Zhang, H. Nikai, and Q. $\mathrm{X}$. Yu, "Adenomatoid odontogenic tumor: Biologic profile based on 499 cases," Journal of Oral Pathology and Medicine, vol. 20, no. 4, pp. 149-158, 1991.

[6] H. P. Philipsen and P. A. Reichart, "Adenomatoid odontogenic tumour: facts and figures," Oral Oncology, vol. 35, no. 2, pp. 125131, 1999.

[7] L. S. Valderrama, "Dentigerous cyst with intracystic adenomatoid odontogenic tumor and complex odontoma.," The Journal of the Philippine Dental Association, vol. 41, no. 3, pp. 35-41, 1988.

[8] A. Warter, G. George-Diolombi, M. Chazal, and A. Ango, "Melanin in a dentigerous cyst and associated adenomatoid odontogenic tumor," Cancer, vol. 66, pp. 786-788, 1990.

[9] Y. Tajima, E. Sakamoto, and Y. Yamamoto, "Odontogenic cyst giving rise to an adenomatoid odontogenic tumor: report of a case with peculiar features," Journal of Oral and Maxillofacial Surgery, vol. 50, no. 2, pp. 190-193, 1992.

[10] V. M. Garcia-Pola, M. G. Garcia, J. S. Lopez-Arranz, and A. H. Zapatero, "Adenomatoid odontogenic tumor arising in a dental cyst: report of unusual case," Journal of Clinical Pediatric Dentistry, vol. 23, no. 1, pp. 55-58, 1998.

[11] M. Bravo, D. White, L. Miles, and R. Cotton, "Adenomatoid odontogenic tumor mimicking a dentigerous cyst," International Journal of Pediatric Otorhinolaryngology, vol. 69, no. 12, pp. 1685-1688, 2005.

[12] Y. K. Chen, I. Y. Hwang, J. Y. Chen, W. C. Wang, and L. M. Lin, "Adenomatoid odontogenic tumour arising from a dentigerous cyst: a case report," International Journal of Pediatric Otorhinolaryngology Extra, vol. 2, pp. 257-263, 2007.

[13] C. F. W. Nonaka, L. B. de Souza, and L. B. Quindere, "Adenomatoid odontogenic tumour associated with dentigerous cystunusual case report," Revista Brasileira de Otorrinolaringologia, vol. 73, pp. 135-137, 2007.

[14] J. E. Leon, G. M. Mata, E. R. Fregnani et al., "Clinicopathological and immunohistochemical study of 39 cases of adenomatoid 
odontogenic tumour: a multicentric study," Oral Oncology, vol. 41, no. 8, pp. 835-842, 2005.

[15] H. P. Philipsen, P. A. Reichart, C. H. Siar et al., "An updated clinical and epidemiological profile of the adenomatoid odontogenic tumour: a collaborative retrospective study," Journal of Oral Pathology and Medicine, vol. 36, no. 7, pp. 383-393, 2007.

[16] D. R. Gadewar and N. Srikant, "Adenomatoid odontogenic tumour: tumour or a cyst, a histopathological support for the controversy," International Journal of Pediatric Otorhinolaryngology, vol. 74, no. 4, pp. 333-337, 2010.

[17] R. S. Buch, W. Coerdt, and U. Wahlmann, "Adenomatoid odontogenic tumor in calcifying odontogenic cyst," Mund-, Kiefer- und Gesichtschirurgie, vol. 7, no. 5, pp. 301-305, 2003.

[18] W. Zhang, Y. Chen, N. Geng, D. Bao, and M. Yang, "A case report of a hybrid odontogenic tumour: ameloblastoma and adenomatoid odontogenic tumour in calcifying cystic odontogenic tumour," Oral Oncology Extra, vol. 42, no. 9, pp. 287-290, 2006.

[19] V. Jivan, M. Altini, S. Meer, and F. Mahomed, "Adenomatoid odontogenic tumour (AOT) originating in a unicystic ameloblastoma : a case report," Head and Neck Pathology, vol. 1, no. 2, pp. 146-149, 2007.

[20] H. P. Philipsen, N. Samman, I. W. Ormiston, P. C. Wu, and P. A. Reichart, "Variants of the adenomatoid odontogenic tumor with a note on tumor origin," Journal of Oral Pathology and Medicine, vol. 21, no. 8, pp. 348-352, 1992.

[21] J. Asaumi, Y. Yanagi, and H. Konouchi, "Assessment of MRI and dynamic contrast enhanced MRI in the differential diagnosis of adenomatoid odontogenic tumor," European Journal of Radiology, vol. 51, no. 3, pp. 252-256, 2004.

[22] M. Toida, I. Hyodo, T. Okuda, and N. Tatematsu, "Adenomatoid odontogenic tumor: report of two cases and survey of 126 cases in Japan," Journal of Oral and Maxillofacial Surgery, vol. 48, no. 4, pp. 404-408, 1990.

[23] B. R. Mendis and D. G. MacDonald, "Adenomatoid odontogenic tumour: a survey of 21 cases from Srilanka," The International Journal of Oral \& Maxillofacial Surgery, vol. 19, no. 3, pp. 141$143,1990$. 


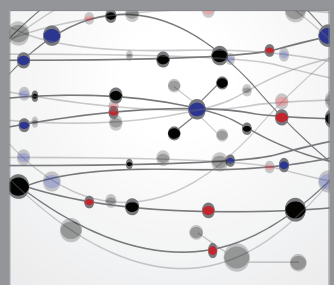

The Scientific World Journal
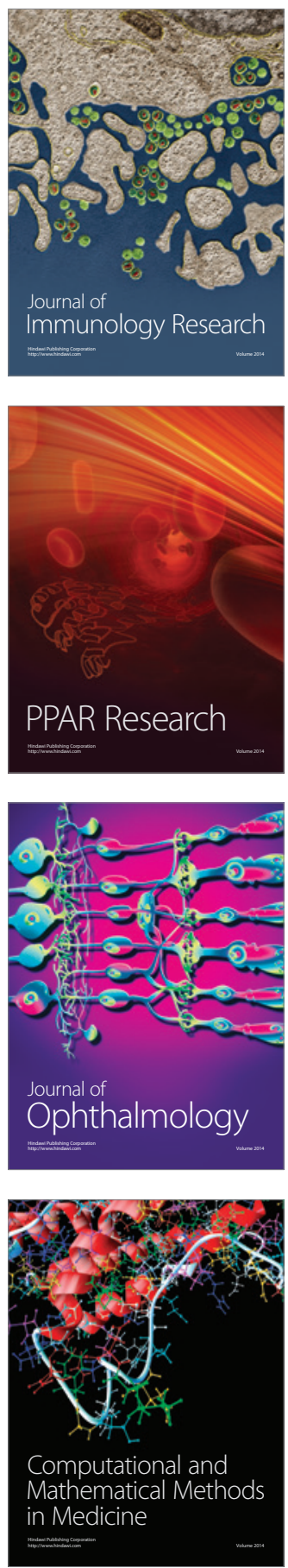

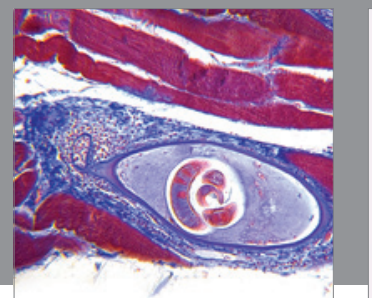

Gastroenterology

Research and Practice
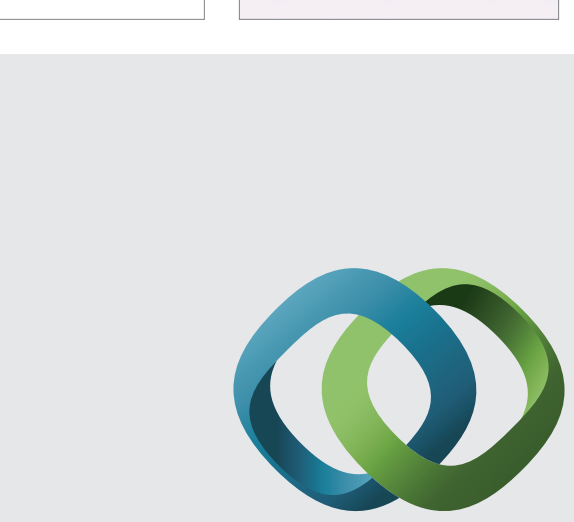

\section{Hindawi}

Submit your manuscripts at

http://www.hindawi.com
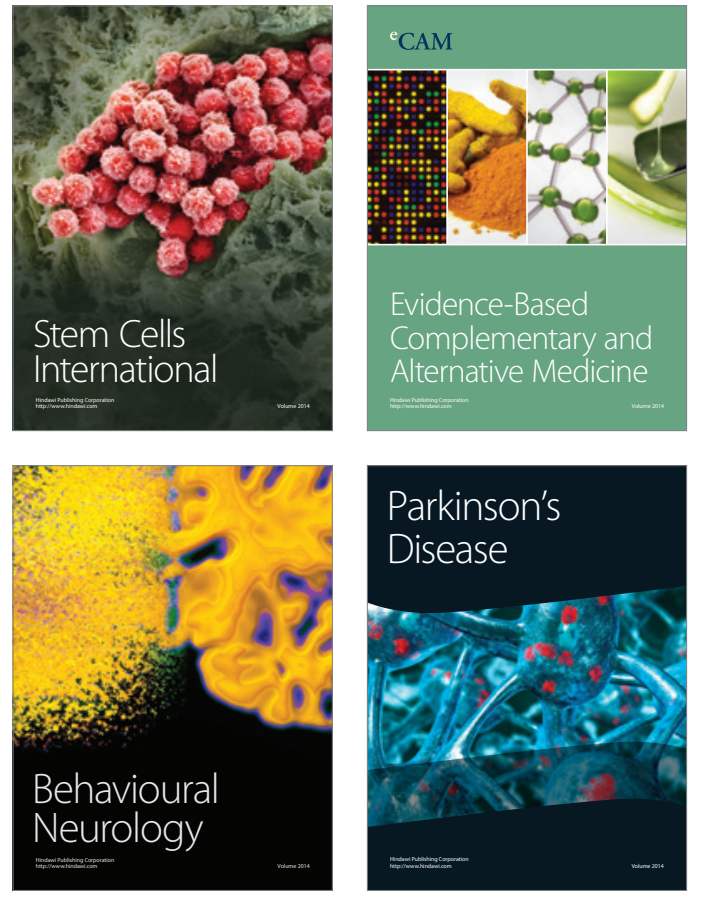
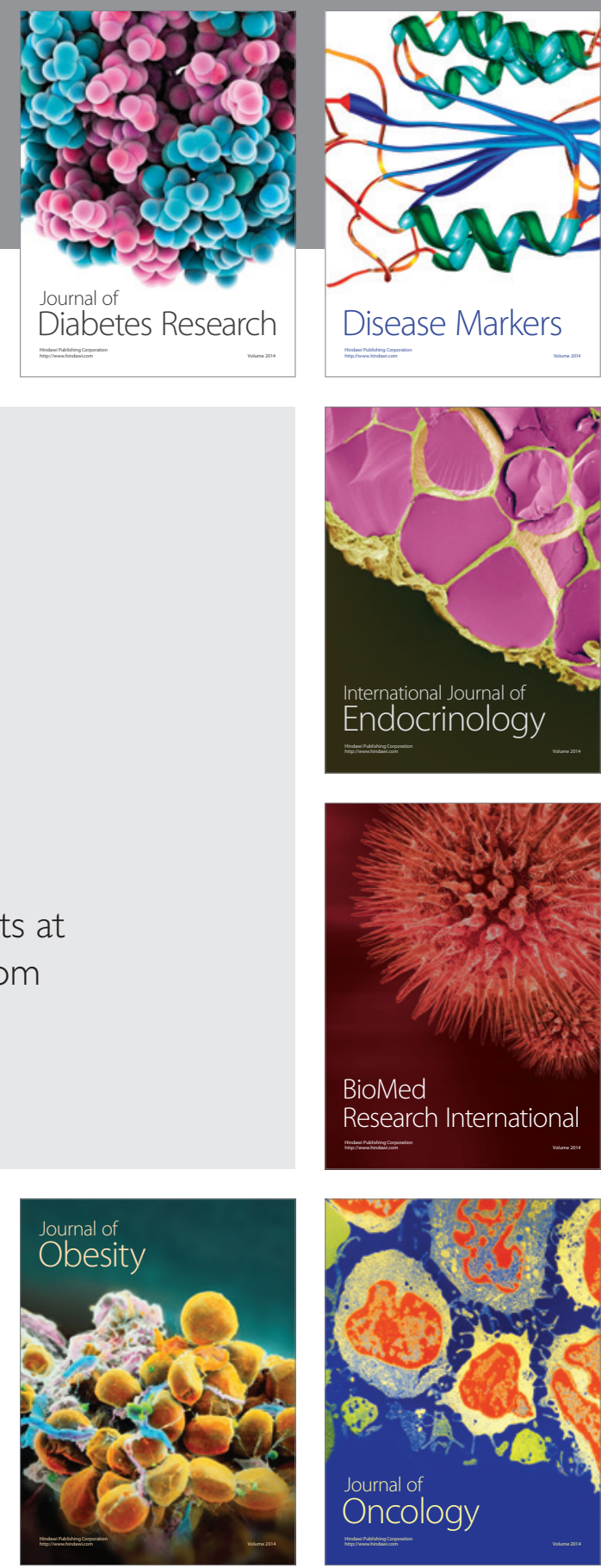

Disease Markers
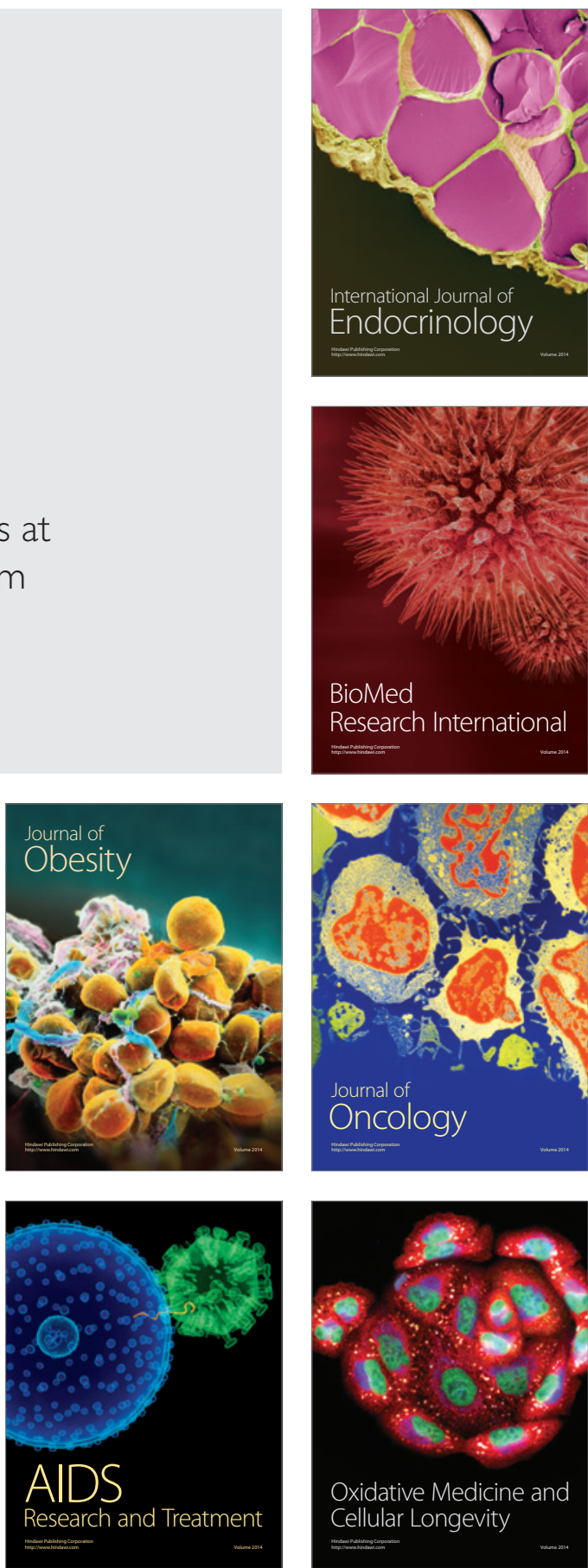